\title{
A Review of Biochemical Markers for Early Diagnosis of Alzheimer's Disease
}

\author{
Meha Fatima Aftab and Rizwana S. Waraich \\ Department of Molecualr Medicine, \\ Institute of Chemical and Biological Sciences, Karachi, Pakistan
}

Received 2012-08-20, Revised 2012-09-01; Accepted 2012-09-04

\begin{abstract}
Alzheimer's disease, the most prevalent type of dementia, affects the life of elderly, to such extent that it impairs the ability to perform routine functions as well. The impairment of normal functions not only affects the patients but the family members as well. It is difficult to make a definitive diagnosis; hence some clinical and psychological tools are used to diagnose the disease. MRI can be used effectively in this regard as well. However in the recent years, much work has been done to devise a biochemical marker which can be effective for definitive and early diagnosis. Amyloid beta and CSF tau proteins have been the most successful by far in confirming the Alzheimer's disease pathology. Other biomarkers such as neuronal markers are also important as they particularly show the pathology in brain. It is recommended by National Institute on Aging to include amyloid beta and markers of neuronal pathology in tools for clinical diagnosis of the disease; even so, more work is required in this regard. It is expected that in future, these markers will be essential to diagnose the disease.
\end{abstract}

Keywords: Amyloid Beta, Neuronal Markers, CSF Tau, Serum p97, Senile Plaques

\section{INTRODUCTION}

In 2001, eleven million people suffered from Alzheimer's disease worldwide (Gadit, 2001). However this number has increased tremendously. In United States alone, out of the 5.2 million people over age 65 , suffering from Alzheimer's disease, 3.4 million are women and 1.8 million are men (CG, 2007; Association, 2012). Out of 36 million people with dementia, 28 million still have to receive a diagnosis and hence are unable to receive treatment. Symptoms of the disease appear usually after the age of 60. Earlier diagnosis can help people plan ahead and make decision about future. Additionally, they and their families can receive timely practical information, advice and support. Through an earlier diagnosis they can get access to available drug and non-drug therapies that may help to improve their cognition and enhance their quality of life. Alzheimer's disease is the sixth-leading cause of death (AA, 2012). Alzheimer's disease is $70 \%$ prevalent in all cases of dementia. Researchers predict that global prevalence will increase four folds by 2050 .
According to another report every $71 \mathrm{sec}$, someone develops Alzheimer's disease. And the rate doubles roughly every 10 years after age 65 (WAD, 2011).

Alzheimer's disease is one of the underlying causes of dementia. Dementia is the term used to indicate impaired brain functions and encompass symptoms like memory loss, confusion, difficulty in performing routine tasks, loss of intellectual functions and impaired judgment. But, this condition is a symptom of many underlying neurological disorders including Alzheimer's disease, vascular dementia (Strokes and TIA's), Dementia with Lewy Bodies (DBL), Parkinson's disease, Frontotemporal Dementia (FTD), Normal-Pressure Hydrocephalus (NPH) and Delirium/Depression. Alzheimer disease is the most prevalent underlying cause of dementia and is clinically evident when there is gradual loss of higher brain functions including change in behavior and mood. The symptoms may progress to disorientation and aphasia (difficulty in language), indicating cortical dysfunction, agnosia (impairment in recognizing object and people), apraxia (impaired motor Corresponding Author: Meha Fatima Aftab, Department of Molecualr Medicine, Institute of Chemical and Biological Sciences, Karachi, Pakistan 
function) and significant of all, memory impairment. With disease progression patients suffer disability and immobility as well. The brain of such patients shows gross cortical atrophy with compensatory ventricular enlargement. The morphologic features include Neurofibrillary Tangles (NFTs), neuritic (senile) plaques (SPs), neuronal loss and amyloid angiopathy. Neurofibrillary tangles are filamentous bundles in cytoplasm of the neurons displacing or encompassing nucleus. In the pyramidal cells, they appear as 'flame' while in rounder cells they appear as 'globos tangles' (Frosch et al., 2010). Senile (neuritic) plaques present outside the neuron, appear as spherical bodies bearing dilated and tortuous neuritic processes around an amyloid beta core which contains some abnormal proteins like amyloid beta plaques which are derived through the processing of Amyloid Precursor Protein (APP) (Frosch et al., 2010; Harvey et al., 2006). The aggregates of amyloid beta obtained from processing of APP are difficult to degrade which consequently activate inflammatory cascade that lead to oxidative injury and alterations in phosphorylation (Frosch et al., 2010). Familial causes or genetic mutations involved in disease pathology include mutations on chromosomes 21, 14 and 1. Risk factors for $\mathrm{AD}$ are advanced age, lower intelligence, small head size, history of head trauma and female gender (Cummings et al., 1998; Yaari and Corey-Bloom, 2007).

\subsection{Biochemical Markers for Early Diagnosis of Alzheimer's Disease 1.1.1. Amyloid Beta}

Amyloid beta comprises of $36-43$ amino acids that is derived from the Amyloid precursor protein. It has many isoforms including 1,14,15,16 and 37, 38 and 39, 40, 42. Some functions of amyloid beta have been discovered including protection against oxidative stress (Zou et al., 2002; Baruch-Suchodolsky and Fischer, 2009), activation of JNK and MAPK enzymes (Bogoyevitch et al., 2004), regulation of cholesterol transport (Yao and Papadopoulos, 2002), enhancement of synaptic plasticity and memory (Puzzo and Arancio, 2012.). It also functions as a transcription factor (Maloney and Lahiri, 2011) and has anti-microbial activity (Soscia et al., 2010) which shows significant association with proinflammatory activity of Amyloid beta. The oligomers of amyloid beta are postulated to cause neurotoxicity by attacking through ligand-binding complexes at specific synapses (Klein, 2012). A 42 levels are decreased in cerebrospinal fluid of Alzheimer's disease patient (Frosch et al., 2010). However, A $\beta-40$ is unchanged. In order to find a biomarker which is more specific, $\mathrm{Ab} 42 / \mathrm{AB} 40$ ratio was calculated and found to be useful in early and clinical phases of Alzheimer's disease. Ratio with other isoforms of Amyloid Beta that are 14, 15, 16 and 37, 38 and 39 are also useful (Frosch et al., 2010). Autopsies of Alzheimer's disease patients revealed the sensitivity of $A \beta 1-42$ to be $79 \%$ and specificity to be $61 \%$ while assessing a diagnostic discrimination of $\mathrm{AD}$ from NADD(non-Alzheimer's dementia) (Roher et al., 2009). $\mathrm{A} \beta-42$ is also found elevated in plasma of Familial Alzheimer Disease (FAD) Mutation Carriers (MCs) and this level might decline with disease progression before developing overt dementia. The ratio of $A \beta 42$ to $A \beta 40$ was also found reduced in the CSF of non demented MCs (mutation carriers) (Ringman et al., 2008).

\subsection{CSF-Tau}

CSF-tau, a microtubule-associated protein, is although sensitive, indicating acute injury following plaque formation, but high CSF-tau is also present in vascular dementia, resulting in a lower specificity. Increased CSF-tau is present during the whole course of the disease in Alzheimer's disease which suggests that it may be present before the onset of clinical dementia (Andreasena et al., 1998; Zetterberga et al., 2003; Arai et al., 1995; Mattsson et al., 2009). Tau is also helpful in discriminating patients of Alzheimer's disease with frontotemporal dementia ${ }^{26}$. Moreover, elevations of $\mathrm{t}$-tau (total tau) and p-tau (phosphorylated tau) 181, the isoforms of Tau protein, are sensitive indicators of presymptomatic disease (Ringman et al., 2008). CSF Ptau231P is found to serve as an in vivo surrogate biomarker of neurofibrillary pathology in Alzheimer's disease (Buerger et al., 2006).

\subsection{Combination of Amyloid Beta and CSF Tau}

Abnormal levels of the CSF tau and amyloid beta proteins in the cerebrospinal fluid are observed in individuals with known Alzheimer's disease and thus these two proteins have been investigated for their diagnostic utility (CCHP, 2011). CSF P-tau is 83\% sensitive and hence can be used to diagnose acute injury and is $75 \%$ specific reflecting its remarkable association with the disease, while $A \beta-42$ has a sensitivity and specificity of 93\% (Zetterberga et al., 2003; BRLS, 2011; Karolinska, 1998). An optimal cutoff of 234 pg $\mathrm{mL}^{-1}$ for total tau has $85 \%$ sensitivity and $84 \%$ specificity (BRLS, 2011) while an optimal cutoff of $361 \mathrm{pg} \mathrm{mL}^{-1}$ has sensitivity and specificity of 72 and $69 \%$ respectively for distinguishing Alzheimer's disease from Frontotemporal Dementia (FTD) and Dementia with Lewy Bodies (DLB) (BRLS, 2011). There is an association of $A \beta-42$ abnormalities with increased rate of cognitive decline, disease progression and risk of conversion to Alzheimer's disease. This association was not found for tau 
abnormalities in CSF. Abnormal levels for A $-42(<495$

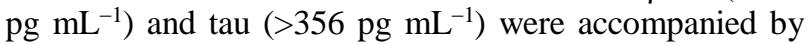
increased (but imprecise) risks for progression to Alzheimer's disease (BRLS, 2011).

Mild Cognitive Impairment, a brain-function syndrome is related to aging, but does not interfere with routine tasks. The period when memory loss is significant in MCI, suggest conversion of MCI to Alzheimer's disease. Pathological levels of $A \beta-42$, total tau and phosphorylated tau (p-tau) that are $\geq 2$ in CSF biomarkers predict MCI (Mild cognitive Impairment) conversion to $\mathrm{AD}$ and indicate the stable form of MCI (Parnettia et al., 2005; Andreasen, 2000). Studies have shown that for the discrimination of AD from ND (nondemented) control subjects, measurement of a set of markers including A $\beta 1-42$, ApoA1 and HPX improved diagnostic performance over that obtained by measurement of A $1-42$ alone $^{33}$. Ratio of phosphorylated tau to $A \beta-42$ is significantly increased in patients with $\mathrm{AD}$ and has high diagnostic accuracy in distinguishing patients with Alzheimer's disease from healthy control subjects with sensitivity of $86 \%$ and specificity of $97 \%$. CSF ratio of phospho-tau to A $\beta-42$ is more useful and can be recommended as an aid for evaluating individuals suspected of dementia due to Alzheimer's disease (Karolinska, 1998; Maddalena et al., 2003; Sobow et al., 2004; Blennow et al., 2001; Humpel, 2011).

\subsection{Amyloid-Beta Derived Difusible Ligands}

Proteins named Amyloid Beta-Derived Diffusible Ligands (ADDLs) are known to have a negative effect at the synapse. The ADDLs contribute to the buildup of amyloid beta protein plaques and this is thought to have a direct effect on cells that store memories and process information. ADDLs are so small (20,000 times finer than a human hair) and so diluted that, up till now, they have been impossible to find in the living. So, a method has been devised using Amyloid beta-derived diffusible ligands in which both gold and magnetic nanoproteins that bind to them are studied. Some of the nanoproteins also bind to a section of DNA which acts like a biochemical barcode. This can then be read in order to detect where ADDLs are concentrated. By using a magnet, the ADDLs attached to the magnetic nanoproteins, can be removed (Kennard, 2005).

\subsection{CSF Monoamine Metabolites}

The CSF monoamine metabolites have been studied to a great extent, but the results are still confusing. At autopsy there is a marked decrease in total brain norepinephrine, serotonin and dopamine, but in vivo measures of the metabolites do not show consistent decreases. Most studies show normal levels, some show reductions, while one study reports an increase in norepinephrine metabolites (Boller, 2002). F2-isoprostane, an oxidative stress marker, is found to be elevated in the CSF of preclinical FAD MCs (Familial Alzheimer's Disease mutation carriers) and indicates that oxidative stress occurs following mis metabolism of amyloid precursor protein (Ringman et al., 2008).

\subsection{Plasma Biomarkers}

The markers detected in plasma, for early diagnosis of Alzheimer's disease, have also been studied but have met little success. These include alpha- 2 macroglobulin and complement factor $\mathrm{H}$, which show increased expression. These are also present in senile plaques. Similarly alpha-1antitrypsin and alpha-1antichymotrpsin and decrease levels of ApolipoproteinA1 in blood plasma and serum are some markers which need to be studied further (Bauer et al., 1991; Strohmeyer et al., 2002; Maes et al., 2006; Matsubara et al., 1990; Liebermann et al., 1995).

\subsection{Inflammatory Markers}

An inflammatory response in Alzheimer's disease has been observed that includes complement activation, elevated C-Reactive Protein (CRP), elevated proinflammatory cytokines (including IL-1-beta, IL-6, TNF-

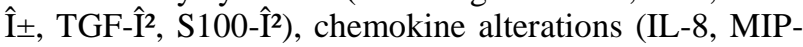
1-alpha, MIP-1-beta, MCP-1) and microglial activation (Molloy et al., 2009). Release of inflammatory mediators occurs in Alzheimer's disease. Several proteins released due to inflammatory process in Alzheimer's disease can be used as biomarker. These proteins were detected in previous studies using ELISA, as well as proteomics approaches (Frosch et al., 2010) The study of cytokines produced during inflammation in Alzheimer's disease, have also shown inconsistent results. For example, CSF interleukin-6 (IL-6) levels have been reported to be increased, decreased, or unchanged in Alzheimer's disease (Pirttila et al., 1994).

\subsection{Transport Binding Proteins}

Albumin, a well known transport binding protein interacts with $A \beta$ in several ways. Albumin is present in Senile Plaques (SP) and prevents the formation of $A \beta$ macroaggregates and protects red blood cell lyses, evidence also indicates that in plasma the majority of $A \beta$ (approximately $89 \%$ ) is bound to albumin. Other $A \beta$ transport proteins include vitamin D-binding protein, which has no known relation to $\mathrm{AD}$ or dementia. Transthyretin, a carrier protein found in serum and CSF, is actually responsible for transporting Thyroxine (T4) and retinol binding protein in a state when it is bound to 
retinol (Ingenbleek and Young, 1994). It is produced by choroid plexus in CSF and by liver in serum. Transthyretin has been found to form stable complexes with $A \beta$ peptides from CSF and may inhibit aggregation of $A \beta$. Apolipoprotein E (ApoE) and ApoJ are the main lipoprotein carriers for $A \beta$. Some studies show a decrease in ApoE isoforms in Alzheimers (Finehout et al., 2007).

\subsection{Urinary Biomarkers}

Urinary neural thread (Neural Thread Protein; NTP), a urinary biomarker test is a sensitive marker reflecting acute injury in the course of disease and $90.1 \%$ specific for diagnosis of probable Alzheimer's disease (BRLS, 2011). Elevated levels of AD7c-NTP (Alzheimer's disease 7c- Neural Thread Protein) isoform can be seen in both cerebrospinal fluid and urine of patients with early or moderately severe Alzheimer's disease and the CSF and urinary levels of AD7c-NTP have been shown to correlate with the severity of dementia. A research show that the newest configuration of the AD7c-NTP assay, called "7c Gold", has greater than $90 \%$ sensitivity and specificity for detecting early Alzheimer's disease. AD7C-NTP concentration in probable AD cases was comparable to cases of early Alzheimer's disease. The data confirmed the specificity of AD7C-NTP as an early biochemical marker of Alzheimer's disease (Boller, 2000; Ghanbari et al., 1998a; Aetna, 1999). It is also found that the competitive ELISA-format AD7C-NTP test in urine is an accurate method for detecting AD7cNTP levels and could be used as a biochemical marker for diagnosis of Alzheimer's disease (Aetna, 1999; Ghanbari et al., 1998b; Levy et al., 2007; Monte and Wands, 2002).

\subsection{Serum and Blood Markers 1.10.1. DHEA- a Blood Test:}

A Hormone, Dehydro-Epiandrosterone (DHEA) is an endogenous steroid hormone (Mo et al., 2006) produced by gonads, brain (Schulman et al., 2007) and adrenal glands and is most abundant of circulating steroid hormones. It is a metabolic intermediate in the biosynthesis of steroid hormones (Scott, 1996). In brain it has been known to be helpful for memory function (Evans et al., 2006). DHEA is present in high levels in the brain and possesses a wide range of biological effects. This hormone was therefore selected for blood test for early diagnosis of Alzheimer's disease. The researchers promoted the production of DHEA, by oxidation, in blood taken from non-Alzheimer's patients. However, it was seen that oxidation of blood from Alzheimer's disease patients did not show increase production of DHEA. Hence it was inferred that reduced production of DHEA by oxidation in blood is related to degree of cognitive impairment found in Alzheimer's disease. Also this test allowed for differential diagnosis of Alzheimer's disease at an early stage (Robert, 2011; Mullins, 2011; Rammouz et al., 2011).

\subsection{Serum Markers}

Hooshmand et al. (2010) studied the relation between serum levels of homocysteine (tHcy) and holotranscobalamin (holoTC), the active fraction of vitamin B12 and risk of developing Alzheimer's. Homocysteine is one of the biochemical tests which is included in the policy for diagnosing Alzheimer's disease (Aetna, 1999).

Serum p97 is a secreted protein which is expressed by amyloid plaque and is associated with reactive microglia in AD. Serum p97 concentration is increased even in mild Alzheimer's disease which makes it useful in the differential diagnosis of Alzheimer's disease from other dementias at early stage. It is known that p97 colocalizes with the transferrin receptor in brain capillary endothelium, which shows the possibility that the soluble p97 protein can cross the blood-brain barrier by interacting with the transferrin receptor. $\mathrm{p} 97$ provides a route for cellular uptake of iron from Fe-citrate that does not require transferrin. It is reported that the concentration of the soluble form of p97 in cerebrospinal fluid is two to four times more than in serum .Therefore, elevation of $\mathrm{p} 97$ in the brain result in the increased serum p97 concentration through the CSF route. As, p97 is localized to microglial cells associated with amyloid plaques in Alzheimer's disease, an elevated serum p97 concentration can be regarded as a substitutive marker of Alzheimer's disease. Despite theoretically being effective it shows difference between clinical diagnosis and classification. Serum p97 concentration reflect nonspecificity and incomplete sensitivity of the laboratory measure, therefore, efficacy is still ambiguous (Kim et al., 2001).

Serum 24S-OH-cholesterol (24S-hydroxycholesterol), a product of cholesterol oxidized enzymatically, is synthesized in the brain. It has found to be an early marker for Alzheimer's disease. Glial fibrillary acidic protein autoantibody (a major fibrous protein of multiple sclerosis plaque) level is suggested to be a late marker for neurodegeneration (Teunissen et al., 2001).

Patients with type II diabetes and Alzheimer's disease express metalloproteases, but their expression is more complex for understanding as these proteins have different pattern of expression between $\mathrm{AD}$ and $\mathrm{AD}$-type II Diabetes patients and among patients and healthy controls (Orlacchio et al., 2008). 
Neuronal and glial derived proteins (such as S100B and NSE) have also been studied in this regard. Glial derived protein S100B (S100 calcium binding protein B) is responsible for, proliferation of melanoma cells, neurite extension stimulation of $\mathrm{Ca}^{2+}$ fluxes, astrocytosis and axonal proliferation, inhibition of PKC-mediated phosphorylation and inhibition of microtubule assembly. In a developing brain it functions as a neurotrophic factor and neuronal survival protein. Hence, serum levels of S100B are studied as a marker for brain functional condition and serum NSE levels reflect morphological status in AD. Serum NSE levels are unchanged while serum levels of S100B are significantly reduced hence a positive correlation between S100B levels and AD severity and a negative correlation between NSE levels and morphological AD has been discovered (Chaves1 et al., 2010).

\subsection{Neuronal Markers}

Contactin, a neuronal membrane protein is known to have a role in communication between neurons and glia and the observed molecular weight and sequence coverage was found consistent with a contactin fragment. Neuronal Pentraxin Receptor (NPR), another neuronal membrane protein was suggested to be involved in the clearance of synaptic debris, although the exact function of this marker was not clear (Finehout et al., 2007) $\alpha 1$ Antichymotrypsin (A1ACT) was observed either increased or unchanged in CSF samples of Alzheimer's disease patients (Matsubara et al., 1990; Harigaya et al., 1995; Pirttila et al., 1994). However some contradictory results show that more studies are required to raise the possibility of A1ACT to be tagged as an effective biomarker (Woon et al., 2007). Alzheimer disease associated protein ADAP, another neuronal marker is found to have three major ALZ-50 subunits including A68.This assay requires ALZ-50 and a rabbit antibody raised against a highly ADAP enriched brain protein fraction. It is suggested to be a helpful diagnostic marker for AD (Ghanbari et al., 1990). Some postmortem studies have shown reduction in cholinergic indices, including Choline Acetyl Transferase (CAT), acetylcholinesterase (AChE) and many cholinergic neurons. Moreover, these changes were correlated with cognitive decline measured clinically (BRLS, 2011). Even then, the results of cholinergic markers studies in the CSF have been disappointing. Measures of AChE have been found normal or reduced, suggesting ambiguity (Boller, 2001).
Protein Kinase $\mathrm{C}$ (PKC) is found in autonomic ganglia and CNS. In autonomic ganglia, it induces excitatory post-synaptic potentials, while in CNS it is also responsible for memory apart from the excitatory function. A recent study suggests the possibility that PKC dependent phosphorylation may be involved in an early stage of plaque formation. Two evidences support this. First, diffuse plaques, which precede the appearance in Alzheimer's disease. Second, the decline of levels of particulate PKC and the reduced PKC-dependent P86 phosphorylation that were found in the cortex of clinically non-demented individuals along with cortical diffuse plaques (Masliah et al., 1991).

\subsection{Genetic Markers}

The protein, ApoE, is present on chromosome 19 in a group with Apolipoprotein $\mathrm{C} 1$ and the Apolipoprotein C2. ApoE has three isoforms, ApoE2, ApoE3 and ApoE4. ApoE- $\varepsilon 3$ is the normal protein, while ApoE- $\varepsilon 2$ and ApoE- $\varepsilon 4$ are the dysfunctional proteins. There is an association between the apolipoprotein E (apoE), ApoE$\varepsilon 4$ genotype and Alzheimer's disease. Several other genotypes of ApoE- $\varepsilon 3$ are discovered i.e., 2, 3 or 4. The ApoE4 allele is found to be over-represented in late onset Alzheimer's. ApoE- $\varepsilon 3$ protect from phosphorylation while ApoE- $\varepsilon 4$ increases the phosphorylation of tau (Karolinska, 1998; Andreasen, 2002) the positive and negative predictive values are inadequate to confirm apoE genotyping as a diagnostic test for Alzheimer's.

CLU gene, present on chromosome 8 produces the protein Clusterin. Clusterin has variety of functions which include regulation of cell lysis mediated by complement, programmed cell death, membrane recycling and cell-cell adhesion. It also acts as complement inhibitor. It binds to and from complexes with, immunoglobulins, complement components, heparin, beta amyloid, bacteria, lipids, leptin, paraoxonase. Clusterin though has variety of functions, but the main function important in Alzheimer's disease is the elimination of the major component of amyloid plaques. CLU gene is also a newly tested gene in this regard, constituting about 9 percent of Alzheimer's cases and CR1, thought to be responsible for about 4 percent of cases. Positive results for the new genetic variations are said to increases a carrier's risk of disease by up to $20 \%$ which is quite less than the 50 to 100 percent increase produced by the APOE marker (Duncan, 2009).

In 1993 Schellenberg demonstrated linkage of the disease to the long arm of chromosome 14. Five mutations in a novel gene from this region (S182) were later identified in several cases of early onset familial 
Alzheimer's Disease (FAD). S-182 was later renamed presenilin 1gene or PS-1gene the resulting protein of which has 467 amino acids and multiple transmembrane domains. Presenilin 1 is a core protein of the resulting presenilin complex. Presenilin is responsible for mediating the regulation of proteolytic event by gamma secretase (and several other proteins), which is responsible for production of beta amyloid, which in the accumulated form, produces amyoid plaques. More than 50 AD-causing mutations up till now have been demonstrated in the PS-1 gene (Andreasen, 2000), the autosomal dominant familial forms of Alzheimer's disease are discovered in three different genetic mutations: Mutations of the Amyloid Precursor Protein (APP) gene on chromosome 21, genes encoding Presenilin 1 (PS1) on chromosome 14 and presenilin 2 (PS2) on chromosome 1. Mutations in the Amyloid Precursor Protein (APP), Presenilin 1 (PS1) and Presenilin 2 (PS2) genes that result in autosomal dominant familial $\mathrm{AD}$ were identified in the early part of the decade (Diaz-Arrastia and Baskin, 2001; Ringman et al., 2008).

Neuronal sortilin receptor gene has also been studied in this regard. The resulting protein is a neuronal apolipoprotein E receptor. Genetic variants of the neuronal sortilin-related receptor with A-type repeats (SORL1, also called LR11 or SORL A) have been found to be risk factors for the development of Alzheimer's Disease (AD). Significant relation between CSF A $\beta$ (142) levels and the SORL1 SNPs 23 and 24 were identified in the AD group (Kim et al., 2011). In addition to known candidate genes, APOE, TOMM40 and one hypothetical gene LOC100129500 partially overlapping APOE; one novel gene, EPC2 and certain other genes were associated with CSF biomarkers that are related to $\mathrm{AD}$. But these findings require further cohort studies (Swaminathan and Shen, 2011).

\subsection{Recent Advances in Alzheimer's Disease Research}

A study by Maria Teresa Ferretti et al. (2012) added new aspects to the pathophysiology of Alzheimer's disease. This study reveals that, before deposition of plaque, some markers of microglial activation are already in process and they are up regulated in the hippocampal region of the transgenic mice studied. These markers include major histocompatibility complex class II (MHC-II), CD40 and inducible Nitric Oxide Synthase (i-NOS). Microglial cells have been observed to be drawn to the region of brain where cells are burdened with amyloid oligomers. Also, neuron-specific cyclooxygenase $2(\mathrm{COX}-2)$ is up regulated specifically in the cells burdened with amyloid beta plaques. The researchers inferred that these inflammatory markers can be used to diagnose early onset Alzheimer's disease (Ferretti et al., 2012). Muller and Zheng in their latest review have also established some important aspects of Amyloid Precursor Protein (APP). The cleavage of APP by $\beta$-secretase (BACE) at the amino terminus of $A \beta$ yields large soluble ectodomians called APPs $\alpha$ and APP $\mathrm{s} \beta$. The primary neuronal cultures have been shown to have high expression of $\beta$-secretase and hence, increased production of $\mathrm{APPs} \beta$ and $\mathrm{AB}$. The AAPs $\beta$ is toxic and is known to bind to death receptor DR6, mediating axon pruning and degeneration (Muller and Zheng, 2011).

\section{CONCLUSION}

Despite extensive work on early diagnosis of Alzheimer's disease, the 2012 Alzheimer's disease facts and figures suggest further research on implementing biochemical markers as necessary tools for diagnosis of the disease. However, these markers are recommended in the new guidelines and amongst the discussed biomarkers, markers for neuronal injury and betaamyloid are suggested for early diagnosis of Alzheimer's disease. These new guidelines have categorized Alzheimer's disease into three stages, the pre-clinical stage of Alzheimer's disease, MCI (Mild cognitive impairment) due to Alzheimer's disease and the last, dementia due to Alzheimer's disease. No biomarker for the pre-clinical stage is recommended; rather more work is required to set any biomarker as the criteria for diagnosis. However, biomarkers are recommended for MCI due to Alzheimer's disease stage but more work is required in this regard too. In future, these biomarkers will be essential to diagnose Alzheimer's disease as suggested by National Institute on Aging (NIA) and Alzheimer's disease Association (Association, 2012).

\section{ACKNOWLEDGEMENT}

My regards and thanks to all the researchers who put their valuable effort in this regard. I am thankful to my professor, Dr. Rizwana S. Waraich for guiding me throughout the study.

\section{REFERENCES}

AA, 2012. Alzheimer's Facts and Figures. Alzheimer's Association. 
Aetna, 1999. Alzheimer's disease: Diagnosis.

Alexopoulos, P., L.H. Guo, M. Kratzer, C. Westerteicher and A. Kurz et al., 2011. Impact of SORL1 single nucleotide polymorphisms on alzheimer's disease cerebrospinal fluid markers. Dementia Geriatric Cognitive Disorders 32: 164-170. DOI: 10.1159/000332017

Andreasen, N., 2000. Search for Reliable Diagnostic Markers for Alzheimer's Disease. 1st Edn., Karolinska Institutet, Stockholm, ISBN-10: 9162840398, pp: 158.

Andreasena, N., E. Vanmechelenb, A. Van de Voordeb, P. Davidssond and C. Hessed et al., 1998. Cerebrospinal fluid tau protein as a biochemical marker for Alzheimer's disease: a community based follow up study. J. Neurol Neurosurg Psychiatry, 64: 298-305.

Arai, D., M. Terajima, M. Miura, S. Higuchi and T. Muramatsu et al., 1995. Tau in cerebrospinal fluid: A potential diagnostic marker in Alzheimer's disease. Annals Neurol., 38: 649-652. DOI: 10.1002/ana.410380414

Association, A., 2012. Alzheimer's disease facts and figures. Alzheimers Dement., 8: 131-68. PMID: 22404854

Baruch-Suchodolsky, R. and B. Fischer, 2009. Abeta40, either soluble or aggregated, is a remarkably potent antioxidant in cell-free oxidative systems. Biochemistry, 48: $\quad$ 4354-70. $\quad$ PMID: 19320465

Bauer, J., S. Strauss and S. Strauss, 1991. Interleukin-6 and alpha-2-macroglobulin indicate an acute-phase state in Alzheimer's disease cortices. FEBS Lett., 285: 111-114. PMID: 1712317

Blennow, K., E. Vanmechelen and H. Hampel, 2001. CSF total tau, Abeta42 and phosphorylated tau protein as biomarkers for Alzheimer's disease. Mol Neurobiol., 24: 87-97. PMID: 11831556

Bogoyevitch, M.A., I. Boehm, A. Oakley, A.J. Ketterman and R.K. Barr, 2004. Targeting the JNK MAPK cascade for inhibition: basic science and therapeutic potential. Biochim. Biophys. Acta, 2: 89-101. PMID: 15023353

Boller, F., 2000. Biological Markers of Alzheimer's Disease. 1st Edn., Springer-Verlag, Berlin, ISBN10: 0387516697, pp: 169.

BRLS, 2011. Biochemical Markers of Alzheimer's Disease.
Buerger, K., M. Ewers, T. Pirttila, R. Zinkowski and I. Alafuzoff et al., 2006. CSF phosphorylated tau protein correlates with neocortical neurofibrillary pathology in Alzheimer's disease. Brain., 129: 30353041. PMID: 17012293

CCHP, 2011. Genetic Testing and Biochemical Markers for the Diagnosis of Alzheimer's.

CG, 2007. Decades-old brain test can diagnose Early Alzheimer's: Study. Pederson Publishing, Inc.

Chaves1, M.L., A.L. Camozzato1, D.E. Ferreira1, I. Piazenski1 and R. Kochhann1 et al., 2010. Serum levels of S100B and NSE proteins in Alzheimer's disease patients. J. Neuroinflammation, 7: 6-6. PMID: 20105309

Cummings, J.L., H.V. Vinters, G.M. Cole and Z.S. Khachaturian, 1998. Alzheimer's disease: etiologies, pathophysiology, cognitive reserve and treatment opportunities. Neurology. 51: 2-17. PMID: 9674758

Diaz-Arrastia, R. and F. Baskin, 2001. New biochemical markers in alzheimer disease. Arch Neurol, 58: 354-356.

Duncan, D.E., 2009. New DNA Markers for Alzheimer's the genetic risk factors could help scientists predict and better understand the disease. Published by MIT,

Evans, G., J. Malouf, R. Huppert and F.J.K. Niekerk, 2006. Dehydroepiandrosterone (DHEA) supplementation for cognitive function in healthy elderly people. Cochrane database of Syst. Rev., PMID: 17054283

Ferretti, M.T., M.A. Bruno, A. Ducatenzeiler, W.L. Klein and A.C. Cuello, 2012. Intracellular A $\beta$ oligomers and early inflammation in a model of Alzheimer's disease. Neurobiol. Aging, 33: 132942. PMID: 21414686

Finehout, E.J., Z. Franck, L.H. Choe and N. Relkin and K.H. Lee, 2007. Cerebrospinal fluid proteomic biomarkers for Alzheimer's disease. Ann Neurol, 61: 120-129. PMID: 17167789

Frosch, M.P., D.C. Anthony and U.D. Girolami, 2010. The Central Nervous System. In: Robbins and Cotran Pathologic Basis of Disease, Robbins, S.L., V. Kumar, A.K. Abbas, R.S. Cotran and N. Fausto (Eds.), Elsevier srl, Philadelphia, ISBN-10: 1416031219, pp: 1313-1317.

Gadit, A.A., 2001. State of mental health in Pakistan. J. Pakistan Med. Assoc.

Ghanbari, H., K. Ghanbari, I. Beheshti, M. Munzar and A. Vasauskas et al., 1998a. Biochemical assay for AD7C-NTP in urine as an Alzheimer's disease marker. J. Clin. Lab. Anal., 12: 285-288. PMID: 9773959 
Ghanbari, H., K. Ghanbari, M. Munzar, M.D. Paul and M.D. Averback, 1998b. Specificity of AD7C-NTP as a Biochemical Marker for Alzheimer's Disease.

Ghanbari, H.A., B.E. Miller, H.J. Haigler, M. Arato and G. Bissette et al., 1990. Biochemical Assay of Alzheimer's Disease-Associated Protein(s) in Human Brain Tissue, Jama, 263: 2907-2910. PMID: 2187102

Harigaya, Y., M. Shoji, T. Nakamura, E. Matsubara and K. Hosoda et al., 1995. Alpha 1-antichymotrypsin level in cerebrospinal fluid is closely associated with late onset Alzheimer's disease. Int. Med., 34: 481484. PMID: 7549128

Harvey, R.A., P.C. Champe, B.D. Fisher, 2006. Lippincott's Illustrated Reviews: Microbiology. 2nd Edn., Lippincott Williams and Wilkins, ISBN-10: 0781782155,pp: 432.

Humpel, C., 2011. Identifying and validating biomarkers for Alzheimer's disease. Trends Biotechnol., 29: 2632. PMID: 20971518

Ingenbleek, Y and V. Young, 1994. Transthyretin (prealbumin) in health and disease: Nutritional Implications. Annu Rev Nutr., 14: 495-533. PMID: 7946531

Kennard, C., 2005. Biochemical 'barcode' may be the Key to Alzheimer's Diagnosis.

Kim, D.K., M.Y. Seo, S.W. Lim, S Kim and J.W. Kim et al., 2001. Serum Melanotransferrin, p97 as a Biochemical Marker of Alzheimer's Disease. Neuropsychopharmacology, 25: 84-90. PMID: 11377921

Kim, S., S. Swaminathan, L. Shen, S.L. Risacher and K. Nho et al., 2011. Genome-wide association study of CSF biomarkers abeta1-42, t-tau and p-tau181p in the ADNI Cohort. Neurology, 76: 69-79. PMID: 21123754

Klein, W.L., 2012. Synaptotoxic amyloid- $\beta$ oligomers: A molecular basis for the cause, diagnosis and treatment of alzheimer's disease? J. Alzheimers Dis., PMID: 22785404

Levy, S., M. McConville, G.A. Lazaro and P. Averback, 2007. Competitive ELISA studies of neural thread protein in urine in Alzheimer's disease. J. Clin. Lab. Anal., 21: 24-33. PMID: 17245761

Liebermann, J., L. Schleissner, K.H. Tachiki and A.S. Kling, 1995. Serum $\alpha 1$-antichymotrypsin level as a marker for Alzheimer-type dementia. Neurobiol. Aging, 16: 747-753. DOI: 10.1016/01974580(95)00056-K
Maddalena, A., A. Papassotiropoulos, B. MullerTillmanns, H.H. Jung and T. Hegi et al., 2003. Biochemical diagnosis of Alzheimer disease by measuring the cerebrospinal fluid ratio of phosphorylated tau protein to beta-amyloid peptide42. Arch Neurol., 60: 1202-6. PMID: 12975284

Maes, O.C., S. Kravitz, Y. Mawal, H. Su and A. Liberman et al., 2006. Characterization of alpha1antitrypsin as a heme oxygenase-1 suppressor in Alzheimer plasma. Neurobiol. Dis., 24: 89-100. PMID: 16887359

Maloney, B. and D.K. Lahiri, 2011. The Alzheimer's amyloid $\beta$-peptide $(\mathrm{A} \beta)$ binds a specific DNA $\mathrm{A} \beta$ interacting domain (ABID) in the APP, BACE1 and APOE promoters in a sequence-specific manner: characterizing a new regulatory motif. Gene, 2: 112. PMID: 21699964

Masliah, E., G.M. Cole, L.A. Hansen, M. Mallorey and T. Albright et al., 1991. Protein kinase c alteration is an early biochemical marker in alzheimer's disease. J. Neurosci., 11: 2759-2767. PMID: 1880547

Matsubara, E., S. Hirai, M. Amari, M. Shoji and H. Yamaguchi et al., 1990. $\alpha 1$-antichymotrypsin as a possible biochemical marker for Alzheimer-type dementia. Annals Neurol., 28: 561-567. DOI: 10.1002/ana.410280414

Mattsson, N., H. Zetterberg, O. Hansson, N. Andreasen and L. Parnetti et al., 2009. CSF biomarkers and incipient alzheimer disease in patients with mild cognitive impairment. JAMA, 302: 385-393. PMID: 19622817

Mo, Q., S.F. Lu and N.G. Simon, 2006. Dehydroepiandrosterone and its metabolites: Differential effects on androgen receptor trafficking and transcriptional activity. J. Steroid Biochem. Mol. Biol., 99: 50-58. PMID: 16524719

Molloy, D.W., B.M. Kucher, S. Jiang and M.P. Rathbone, 2009. A Pilot Study of Inflammatory Markers in Alzheimer's Disease. McMaster University.

Monte, S.M.D.L. and J.R. Wands, 2002. The AD7c-ntp neuronal thread protein biomarker for detecting Alzheimer's disease. Frontiers Biosci., 3: 345-353. PMID: 12214056

Muller, U.C. and H. Zheng, 2011. Physiological functions of APP family proteins. Cold Spring Harbor Laboratory Press, DOI: 10.1101/cshperspect.a006288 
Mullins, K.J., 2011. Blood test for Alzheimer's disease may hit the market soon. Digital J.

Orlacchio, A., C. Massini, R. Tiribuzi, E. Costanzi and G. Makrypidi et al., 2008. P1-396: Identification of biochemical markers for the diagnosis of Alzheimer's disease in patients affected by Type II diabetes. J. Alzheimer's Associat., DOI: 10.1016/j.jalz.2008.05.977

Parnettia, L., A. Lanaria, G. Silvestrellia, E. Saggesea and P. Reboldib, 2005. Diagnosing prodromal Alzheimer's disease: Role of CSF Biochemical Markers. DOI: 10.1016/j.mad.2005.09.022

Pirttila, T., P.D. Mehta, H. Frey and H.M. Wisniewski, 1994. $\alpha 1$-Antichymotrypsin and IL-1 $\beta$ are not increased in CSF or serum in Alzheimer's disease. Neurobiol. Aging, 15: 313-317. DOI: 10.1016/01974580(94)90026-4

Puzzo, D. and O. Arancio, 2012. Amyloid- $\beta$ Peptide: Dr. Jekyll or Mr. Hyde? J. Alzheimers Dis., PMID: 22735675

Rammouz, G., L. Lecanu, P. Aisen and V. Papadopoulos, 2011. A lead study on oxidative stress-mediated dehydroepiandrosterone formation in serum: the biochemical basis for a diagnosis of Alzheimer's disease. J. Alzheimer's Dis., 2: 5-16. PMID: 21335661

Ringman, J.M., S.G. Younkin, D. Pratico, W. Seltzer and G.M. Cole et al., 2008. Biochemical markers in persons with preclinical familial Alzheimer disease, 71: 85-92. PMID: 18509095

Robert, J., 2011. Blood test for Alzheimer's.

Roher, A.E., C.L. Maarouf, L. Sue, Y. Hu and W. Jeffrey et al., 2009. Proteomics-derived cerebrospinal fluid markers of autopsy-confirmed Alzheimer's disease. Biomarkers, 14: 493-501. PMID: 19863188

Schulman, R.A., M.D. Dean and M.D. Carolyn, 2007. DHEA (Dehydroepiandrosterone) is a common hormone produced in the adrenal glands, the gonads and the brain.

Scott, T., 1996. Concise Encyclopedia Biology. 1st Edn., Walter de Gruyter, Berlin, ISBN-10: 3110106612, pp: 1287.

Sobow, T., M. Flirski and P.P. Liberski, 2004. Amyloidbeta and tau proteins as biochemical markers of Alzheimer's disease. Acta Neurobiol. Exp., 64: 5370. PMID: 15190680
Soscia, S.J., J.E. Kirby, K.J. Washicosky, S.M. Tucker and M. Ingelsson et al., 2010. The Alzheimer's disease-associated amyloid beta-protein is an antimicrobial peptide. PLoS One, 5: e9505- e9505. PMID: 20209079

Strohmeyer, R., M. Ramirez, G.J. Cole, K. Mueller and J. Rogers, 2002. Association of factor $\mathrm{H}$ of the alternative pathway of complement with agrin and complement receptor 3 in the Alzheimer's disease brain. J. Neuroimmunol., 131: 135-146. PMID: 12458045

Teunissen, C.E., J. de Vente, H.W. Steinbusch and C. De Bruijn, 2001. Biochemical markers related to Alzheimer's dementia in serum and cerebrospinal fluid. Neurobiol. Aging, 23: 485-508. PMID: 12009495

WAD, 2011. World Alzheimer's Day on Wednesday.

Woon, W.L., A. Cichocki, F. Vialatte and T. Musha, 2007. Techniques for early detection of Alzheimer's disease using spontaneous EEG recordings. Physiol. Meas. 28: 335-347. PMID: 17395990

Yaari, R. and J. Corey-Bloom, 2007. Alzheimer's disease: Pathology and pathophysiology. Semin Neurol. 27: 32-41.

Yao, Z.X. and V. Papadopoulos, 2002. Function of betaamyloid in cholesterol transport: A lead to neurotoxicity. FASEB J., 16: 1677-9. PMID: 12206998

Zetterberga, H., L.O. Wahlundb and K. Blennowa, 2003. Cerebrospinal fluid markers for prediction of Alzheimer's disease. Neurosci. Lett., 352: 67-69. PMID: 14615052

Zou, K., J.S. Gong, K. Yanagisawa and M. Michikawa, 2002. A novel function of monomeric amyloid betaprotein serving as an antioxidant molecule against metal-induced oxidative damage. J. Neurosci., 22: 4833-4841. PMID: 12077180 